\title{
Piperacillin-Tazobactam-Induced Neutropenia: A Case Report
}

\author{
Diana Darwiche ${ }^{\mathrm{a}}$, Katia Iskandar ${ }^{\mathrm{a}}$, Rania Azar ${ }^{\mathrm{a}}$, Rabih Hallit ${ }^{\mathrm{b}}$, \\ Souheil Hallita, b, c, d, e
}

\begin{abstract}
Piperacillin/tazobactam (TZP) is a beta-lactam antibiotic used for the treatment of many infections and is generally considered safe. Neutropenia is a rare but serious adverse effect of TZP. In this study, we present the case of piperacillin/tazobactam-induced neutropenia in an elderly patient, from which clinical data and blood samples were collected. Leucocytes and neutrophils counts were decreased on day 3 after initiating the treatment and worsened on day 5 . We hypothesize that TZP might be able to cause neutropenia in a period shorter than 10 days. The relation between the duration of treatment with TZP and the onset of neutropenia requires additional studies.
\end{abstract}

Keywords: Piperacillin; Tazobactam; Neutropenia

\section{Introduction}

Piperacillin/tazobactam (TZP) is a combination of a semi-synthetic ureidopenicillin and a beta-lactamase inhibitor, with an extended spectrum of action against beta-lactamase producing organisms and an increased activity against Pseudomonas aeruginosa when compared with other penicillins [1]. Its known adverse effects include hypersensitivity reactions, neurotoxicity, hepatotoxicity, electrolyte and acid-base disturbances, and bleeding disorders [1]. In addition, bone marrow suppression is a recognized adverse drug reaction of beta-lactam antibiotics, including neutropenia, thrombocytopenia and rarely hemolytic anemia [1]. A recent systematic review has supported the assumption that piperacillin can also have such adverse effects [2].

Neutropenia is defined as a decrease in the circulating neutrophils in the non-marginal pool to less than 1,500 cells/

Manuscript submitted July 31, 2017, accepted August 22, 2017

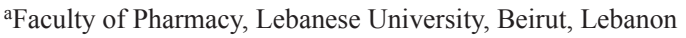

${ }^{b}$ Faculty of Medicine and Medical Sciences, Holy Spirit University of Kaslik, Kaslik, Lebanon

${ }^{\mathrm{c}}$ Faculty of Pharmacy, Saint-Joseph University, Beirut, Lebanon

${ }^{\mathrm{d}}$ Research Department, Psychiatric Hospital of the Cross, PO Box 60096, Jal Eddib, Lebanon

${ }^{e}$ Corresponding Author: Souheil Hallit, Street 8, Building 560, 1st Floor, Biakout, Mount Lebanon, Lebanon. Email: souheilhallit@hotmail.com

doi: https://doi.org/10.14740/jmc2889w $\mathrm{mm}^{3}$ [3]. It is classified as mild, moderate or severe based on the absolute neutrophil count (ANC), with the latter measuring the number of neutrophil granulocytes present in the blood. Mild neutropenia is defined as an ANC of 1,000 - 1,500 cells/ $\mathrm{mm}^{3}$, moderate neutropenia with an ANC of $500-1,000$ cells/ $\mathrm{mm}^{3}$, while severe neutropenia refers to an ANC lower than 500 cells $/ \mathrm{mm}^{3}$ [4]. Most of the neutrophils are contained in the bone marrow, either as mitotically active or post-mitotic mature cells [5].

The reduced number of neutrophils makes patients extremely vulnerable to infection [6] and the risk of bacterial infection is related to both the severity and duration of the neutropenia.

There seems to be a relationship between the duration of TZP treatment and the onset of neutropenia. Most reviewed studies showed that neutropenia rarely develops before 10 days of treatment [7-9]. In one of the published case reports, a patient developed agranulocytosis on day 17 while receiving TZP treatment for an intracranial infection [10]. The mechanism by which TZP causes neutropenia is unclear but it is believed that piperacillin causes proliferation arrest of the myeloid cells [11]. Moreover, a published case report has detected immunologically mediated neutropenia in the presence of $\operatorname{IgG}$ antibodies against penicillins [12].

We report a case of neutropenia that occurred in a patient treated with TZP for pneumonia before 10 days of treatment. Monitoring for hematological parameters is crucial in a patient receiving piperacillin even for a short period.

\section{Case Report}

A 91-year-old man with a history of diabetes and benign prostatic hypertrophy, treated with glimepiride and alfuzosin respectively, was admitted to the intensive care unit (ICU) at the Lebanese Canadian Hospital (Lebanon) in March 2017 with dyspnea. On admission, his temperature was $37{ }^{\circ} \mathrm{C}$, with a blood pressure of $90 / 50 \mathrm{~mm} \mathrm{Hg}$, a pulse of 76 beats per minute and an oxygen saturation of $92 \%$ on room air. A chest X-ray showed a pleural effusion and a right lower lobe pneumonia. He was treated with TZP at a dose of $4.5 \mathrm{~g}$ IV every $8 \mathrm{~h}$. The complete blood count at baseline (day 1) showed a microcytic hypochromic anemia $(\mathrm{Hb}=10.4 \mathrm{~g} / \mathrm{L}, \mathrm{Ht}=32 \mathrm{~g} / \mathrm{L}, \mathrm{GR}=4.24$, $\mathrm{MCV}=76, \mathrm{MCH}=24)$, a white cell count $\left(4,200\right.$ cells $\left./ \mathrm{mm}^{3}\right)$ (46\% neutrophils). On day 2 and 3 , the patient was hemodynamically stable and the dyspnea improved. On day 3 , the pa- 
Table 1. Laboratory Tests Results During Hospitalization

\begin{tabular}{llll}
\hline & Day $\mathbf{1}$ & Day 3 & Day 5 \\
\hline White blood cells count & 4,200 & 2,900 & 3,000 \\
Neutrophils & $48 \%$ & $46 \%$ & $26 \%$ \\
Lymphocytes & $41 \%$ & $42 \%$ & $62 \%$ \\
Eosinophils & $1 \%$ & $2 \%$ & $2 \%$ \\
Monocytes & $10 \%$ & $10 \%$ & $10 \%$ \\
Hematocrit & 32 & 28 & 27 \\
Hemoglobin & 10.4 & 9.1 & 8.9 \\
Red blood cells count & 4.24 & 3.7 & 3.59 \\
Mean corpuscular volume (MCV) & 76 & 75 & 75 \\
Mean corpuscular hemoglobin (MCH) & 24 & 25 & 25 \\
Mean corpuscular hemoglobin concentration (MCHC) & 31.9 & 32.9 & 33.1 \\
Platelets & 212,000 & 171,000 & 148,000 \\
\hline
\end{tabular}

tient developed a leucopenia with a white cell count of 2,900 cells $/ \mathrm{mm}^{3}$ associated with neutropenia (neutrophils $46 \%$ ). On day 5 of treatment, the neutropenia worsened (neutrophils $26 \%$ ) and the white cell count was 3,000 cells $/ \mathrm{mm}^{3}$ (Table 1). TZP was discontinued and the patient received levofloxacin. The white cell count came back to normal 3 days after stopping TZP (data not shown). The sputum culture was negative.

\section{Discussion}

Piperacillin is an aminobenzyl penicillin derivative used for the treatment of infections caused by certain organisms (Pseudomonas aeruginosa, Klebsiella, etc.). Mild infections require a dose of $4-12 \mathrm{~g}$ /day; however, serious infections require a higher dose (12 - $24 \mathrm{~g} /$ day) [13]. In most studies, large cumulative doses are required for the onset of neutropenia, a side effect not commonly reported [1]. Neutropenia is an uncommon but serious adverse event of piperacillin. There have been several previous reports of neutropenia and bone marrow suppression following the use of piperacillin and TZP. A systematic review of studies on the relationship between piperacillin use and neutropenia suggests that the occurrence of neutropenia varies between $0.04 \%$ and $34 \%$ among individuals exposed to this drug [2]. Moreover, a retrospective cohort study in patients treated with piperacillin for osteomyelitis showed that $34 \%$ (14 of 41 ) of patients treated for more than 10 days developed neutropenia. It is of note that cumulative doses of piperacillin administered to neutropenic patients were higher than those administered to non-neutropenic ones [14].

In six previous reports of patients receiving piperacillin to treat osteomyelitis, pneumonia, cystic fibrosis and abscess, neutropenia has been reported 15 days after the start of therapy $[7,9,12]$. In four other published case reports of patients between 19 and 50 years, receiving TZP (dose $=4.5 \mathrm{~g}$ IV every $8 \mathrm{~h}$ ) to treat pancreatic infections, pneumonia, osteomyelitis, neutropenia has been reported $16-25$ days after initiation of treatment $[9,15,16]$. In one of these cases, the patient received metronidazole and amikacin as well. However, metronidazole was unlikely to be the cause because it had been administered $12 \mathrm{~h}$ prior to the appearance of neutropenia and amikacin had never been implicated as a cause of neutropenia [15]. Bone marrow examination in another case detected a maturation arrest of granulocytic cells [9]. After suspecting a TZP-induced bone marrow suppression due to TZP, the medication was discontinued immediately in all cases, with the neutrophil counts recovering completely $4-6$ days after stopping the drug $[9$, $15,16]$.

A retrospective analysis in 38 children with cystic fibrosis (age $=14$ years) receiving TZP courses had detected six cases of fever, malaise, and headache during treatment without signs of acute infection. Out of these 38 cases, three children developed neutropenia between day 11 and day 15 of treatment. These children had received a higher cumulative dose of TZP than in the unaffected group. After discontinuation of TZP, blood cell counts were normalized [17].

Moreover, FDA Adverse Event Reporting System Database (AERS Database) detected 366 cases of hematological disorders (50\% neutropenia and 27\% leucopenia) following piperacillin administration, with neutropenia occurring on days $5-15$ in 62 of these cases.

Bone marrow toxicity, mainly neutropenia, has been described as an uncommon secondary effect of TZP, and is usually related to large cumulative dose in many published cases [9, 15-17]. In our 91-year-old patient, neutropenia occurred without evidence of cumulative dose administered. Thus, we hypothesize that the neutropenia can be related to the medication itself and not to the cumulative dose. Further research are needed to evaluate this correlation.

\section{Conclusion}

Bone marrow suppression, especially neutropenia, is a serious adverse effect of TZP, which should be kept in mind while treating patients with this drug. Neutropenia occurred 3 days 
after the start of piperacillin treatment, despite being reported after 10 days of treatment in previous papers. Therefore, the association between the duration of treatment with TZP and the onset of neutropenia requires additional studies.

\section{Conflicts of Interest}

The authors have nothing to disclose.

\section{Funding}

The authors received no funding from an external source.

\section{References}

1. Kucers A, Crowe S, Grayson M, Hoy J. The use of antibiotics: a clinical review of antibacterial. Antifungal and Antiviral Drugs. 1997:1214.

2. Scheetz MH, McKoy JM, Parada JP, Djulbegovic B, Raisch DW, Yarnold PR, Zagory J, et al. Systematic review of piperacillin-induced neutropenia. Drug Saf. 2007;30(4):295-306.

3. Strom BL, Carson JL, Schinnar R, Snyder ES, Shaw M. Descriptive epidemiology of agranulocytosis. Arch Intern Med. 1992;152(7):1475-1480.

4. Haddy TB, Rana SR, Castro O. Benign ethnic neutropenia: what is a normal absolute neutrophil count? J Lab Clin Med. 1999;133(1):15-22.

5. Smith C. Production, distribution, and fate of neutrophils. Williams hematology. 2006:9.

6. Palmblad J, Nilsson CC, Hoglund P, Papadaki HA. How we diagnose and treat neutropenia in adults. Expert Rev Hematol. 2016;9(5):479-487.
7. Gerber L, Wing EJ. Life-threatening neutropenia secondary to piperacillin/tazobactam therapy. Clin Infect Dis. 1995;21(4):1047-1048.

8. Rye PJ, Roberts G, Staugas RE, Martin AJ. Coagulopathy with piperacillin administration in cystic fibrosis: two case reports. J Paediatr Child Health. 1994;30(3):278-279.

9. Khan F. Severe neutropenia secondary to piperacillin/ tazobactam therapy. Indian Journal of Pharmacology. 2005;37:192.

10. He ZF, Wu XA, Wang YP. Severe bone marrow suppression and hepatic dysfunction caused by piperacillin/tazobactam. Scand J Infect Dis. 2013;45(11):885-887.

11. Neftel KA, Hauser SP, Muller MR. Inhibition of granulopoiesis in vivo and in vitro by beta-lactam antibiotics. J Infect Dis. 1985;152(1):90-98.

12. Ruiz-Irastorza G, Barreiro G, Aguirre C. Reversible bone marrow depression by high-dose piperacillin/tazobactam. Br J Haematol. 1996;95(4):611-612.

13. Winston DJ, Murphy W, Young LS, Hewitt WL. Piperacillin therapy for serious bacterial infections. Am J Med. 1980;69(2):255-261.

14. Peralta FG, Sanchez MB, Roiz MP, Pena MA, Tejero MA, Arjona R. Incidence of neutropenia during treatment of bone-related infections with piperacillin-tazobactam. Clin Infect Dis. 2003;37(11):1568-1572.

15. Kumar A, Choudhuri G, Aggarwal R. Piperacillin induced bone marrow suppression: a case report. BMC Clin Pharmacol. 2003;3:2.

16. Suntur BM, Kuscu F, Hava K, Dalkiran PA. [Piperasilin/ tazobaktama bagli notropeni gelisen bir olgu sunumu]. Ege Tip Dergisi. 2015;54.

17. Reichardt P, Handrick W, Linke A, Schille R, Kiess W. Leukocytopenia, thrombocytopenia and fever related to piperacillin/tazobactam treatment - a retrospective analysis in 38 children with cystic fibrosis. Infection. 1999;27(6):355-356. 